\title{
Spectroscopy of the open cluster Blanco ${ }^{\star}$
}

\section{II. $\mathrm{H} \alpha$ emission as an indicator of relative age}

\author{
P.M. Panagi ${ }^{1,2}$ and M.A. O'Dell ${ }^{2,3}$ \\ 1 Joint Center for Mesoscale Meteorology, Universty of Reading, Reading, UK \\ 2 Visiting Fellow, Astronomy Centre, Univ. Sussex, Falmer, Brighton, BN1 9QH, UK \\ 3 Dept. Physics \& Astronomy, Univ. St. Andrews, North Haugh, Fife, KY16 9SS, Scotland
}

Received September 13, 1995; accepted May 22, 1996

\begin{abstract}
We present the results of $\mathrm{H} \alpha(6562 \AA)$ and Li I $(6708 \AA)$ observations of 114 low-mass stars of the young open cluster Blanco 1. We also present observations of 30 stars in $\mathrm{Ca} \mathrm{II}(\mathrm{K})$. This work extends the first Blanco 1 spectroscopic study of Panagi et al. (1994).

From a sample of four well-studied clusters, including Blanco 1, we find that the fraction of $\mathrm{H} \alpha$ emission-line stars amongst $\mathrm{K}$ dwarfs is a good indicator of relative age, with a smaller fraction indicative of older age. Blanco 1 shows a relatively small fraction of emitters, inconsistent with previous age estimates for the cluster. We estimate the cluster age to to be $90 \pm 25 \mathrm{Myr}$, slightly older than the Pleiades. The method is shown to be more sensitive to age than lithium and a useful alternative to other age measurement techniques.

The variation of $\mathrm{H} \alpha$ with $(B-V)$ is similar to that observed in the older solar neighbourhood dwarfs, suggesting that, at least for the absorption-line stars, the contribution of stellar rotation to the equivalent width is unclear.

We combine both spectroscopy and photometry to revise cluster membership and give accurate positions for all these stars.
\end{abstract}

Key words: Galaxy: open clusters and associations: blanco 1 - stars: activity — stars: chromospheres stars: late-type

\section{Introduction}

The young galactic cluster Blanco 1 is a loose aggregate of stars lying beyond the bright B-type star $\zeta$ Sculptoris. It was first identified by Blanco (1949) who noticed the area

Send offprint requests to: P.M. Panagi

* Tables A1 and A2 are available at the CDS via anonymous ftp 130.79.128.5 or http://cdsweb.u-strabg.fr./Abstract.html contained a significantly higher concentration of A0-type stars than the average value for similar galactic latitudes.

Since its discovery the cluster has been subject to considerable photo-electric and photographic multi-colour photometry, e.g. Westerlund (1963), Epstein (1968), Eggen (1970, 1972), Perry, et al. (1978), Lyngå \& Wramdemark (1984), Abraham de Epstein \& Epstein (1985) and Westerlund et al. (1988).

Dolidze (1959) reported the presence of $\sim 70$ stars in the cluster region (magnitude range: $8 \leq V \leq 16$ ) that showed $\mathrm{H} \alpha$ in emission. These observations have since been questioned by Bond (1972) who found no stars exhibiting bright $\mathrm{H} \alpha$ although he considered it possible that very faint emission-line stars may exist between $V=14$ and 16 .

In a recent paper, Panagi et al. (1994) (hereafter Paper I), presented the first detailed spectroscopic survey of the low-mass component of the Blanco 1 cluster. The observations covered 115 stars from the survey of Abraham de Epstein \& Epstein (1985), taking in the chromospheric lines of $\mathrm{Ca} \mathrm{II}(\mathrm{H}, \mathrm{K})$, Ca II (I-R) triplet and $\mathrm{H} \alpha$. This preliminary survey found that a very low proportion of stars showed $\mathrm{H} \alpha$ emission and all lie approximately in the magnitude range suggested by Bond.

Emission and absorption in chromospheric lines acts as an important proxy for the dynamo that is thought to give rise to heating in the chromosphere. This in turn depends on the rotation rate and ultimately on the stellar age, so that chromospheric studies can give important clues as to a cluster's age. Stars that show $\mathrm{H} \alpha$ emission are generally more active than absorption-line stars. Studies of chromospheric activity in young clusters such as the Pleiades (Prosser et al. 1991), the Hyades (Stauffer et al. 1991) and $\alpha$ Persei (Prosser 1992) have shown that the mean $\mathrm{H} \alpha$ emission strength in the late K and M-type dwarfs declines with age. An earlier study of the Pleiades by Stauffer \& Hartmann (1987) showed that a strong correlation exists between $\mathrm{H} \alpha$ emission and rotation, for stars earlier 
than about M0. This, and other surveys, led Stauffer et al. (1991) to suggest that the age-dependence of $\mathrm{H} \alpha$ emission could be calibrated to measure relative cluster ages. A paucity of $\mathrm{H} \alpha$ emitters in a cluster's late-type population is therefore an indication of greater age. By comparing the ratio of the number of emitters amongst the $\mathrm{K}$ dwarf population for a variety of clusters, we attempt to calculate a new age for Blanco 1 (as first described in O'Dell 1995).

Previous age estimates, derived by fitting isochrones to the cluster's colour-magnitude diagram, suggested an age of $~ 50$ Myr (Perry et al. 1978; Abraham de Epstein \& Epstein 1985; Westerlund et al. 1988) i.e. of comparable age to the Pleiades and $\alpha$ Persei clusters.

Following Paper I, we extended the lithium survey of the cluster. Although recognised as an indicator of youth, an understanding of the lithium depletion process is far from established and ages for individual stars obtained from lithium studies are subject to doubt. Nevertheless as a check we decided to compare the spread of lithium abundances with colour, for Blanco 1, with those of other young clusters to recover an approximate age and compare to that obtained from the relative number of $\mathrm{H} \alpha$ emitters amongst the $\mathrm{K}$ dwarfs.

Finally we present accurate positions for all the stars surveyed by Abraham de Epstein \& Epstein and revise cluster membership, incorporating all the proposed cluster members of Westerlund et al. (1988).

\section{Astrometry and observations}

\subsection{Stellar positions}

To date, the most extensive survey of the cluster is that published by Abraham de Epstein \& Epstein (1985) who were the first to examine stars fainter than $V \simeq 12.6$. Their photometric survey encompasses a considerable proportion of the late-type population of the cluster, notably the G and K-dwarfs. Westerlund et al. (1988) studied 130 stars in a much larger region surrounding the known centre of the cluster. This survey was restricted to stars brighter than $V \simeq 12.6$ and therefore Abraham de Epstein \& Epstein (1985) remains the primary data source for the late-type stars. Neither of these papers presents accurate astrometry, although Abraham de Epstein \& Epstein (1985) give $X$ and $Y$ plate co-ordinates for 262 stars taken from the photographic plate used in their survey.

As all the observed stars were from the Abraham de Epstein \& Epstein survey, we transformed all the $X$ and $Y$ co-ordinates to $(\alpha, \delta)$ (B1950.0) using the APM facility at the Royal Greenwich Observatory (Irwin \& McMahon 1992) and cross-checked the target stars with the appropriate plate of the Schmidt southern sky survey.

The epoch B1950.0 positions of all the objects that we consider to be members are given in Table A1 (available electronically) (the criteria for membership is discussed in Sect. 3.1). Some stars were not recovered by the APM.
These invariably lay in regions close to bright stars (e.g. within the diffraction ring of $\zeta$ Sculptoris). Others were not detected as stellar objects due to the shape of their intensity profile and were classified non-stellar. Although a few of these were extragalactic objects the majority, on visual inspection, were found to be close stellar pairs that were unresolved by the scan. Those stars whose positional accuracy is in doubt are annotated accordingly in Table A1.

This detailed study of the Abraham de Epstein \& Epstein sample revealed some interesting anomalies. The 'stars' ZS 51, 59, 86 and 87 are in fact elliptical galaxies. Close examination of the Schmidt plate reveals that stars of comparable brightness exhibit diffraction spikes whereas these particular objects exhibit characteristic galactic haloes. The finding charts show ZS 250 and 255 to be the same object. This is confirmed by examining the photometry of Abraham de Epstein \& Epstein (1985) which is virtually identical for these stars. Table A2 in the appendix lists the positions of all the non-members, derived either from previous publications, inspection of the Schmidt plate, photometry or spectroscopy.

\subsection{Observations}

The observations were taken between the $24^{\text {th }}$ and $27^{\text {th }}$ October 1993, using the AUTOFIB (Sadler et al. 1991) fibre optic feed from the prime focus of the AAT to the RGO spectrograph, with $25 \mathrm{~cm}$ camera, Tektronix $1024 \times 1024$ pixel CCD. The 1200R grating used gave a resolving power $\lambda / \Delta \lambda \sim 14000$. In all, the 64 available fibres present a $38^{\prime \prime}$ field, with the added constraint that stars in a given exposure must be within 2 magnitudes of each other. As the cluster is spread out over some 1.5 degrees, only 16 stars at most were used for any one exposure. The remaining fibres were used to obtain an accurate measure of the sky background. Stellar positions need to be accurate to 0."5, because of the small entrance apertures to the fibres. Exposure times were $1500 \mathrm{~s}$ and $6000 \mathrm{~s}(2 \times 3000 \mathrm{~s})$ at $\mathrm{H} \alpha$ and Ca II, for stars with magnitude $B \geq 17$, and $600 \mathrm{~s}$ and 1200 s respectively for stars with $B \leq 17$.

The standard CCD reduction methods of bias subtraction, flat-fielding, scattered light and sky subtraction, fibre transmission, and wavelength calibration were performed with the STARLINK software collection (Lawden 1995).

The spectral range centred on $\mathrm{H} \alpha$ covered approximately the region $6300-6800 \AA$, encompassing also the Li I $(6708 \AA)$ line. For our subsequent analysis, we only required $\mathrm{H} \alpha$ equivalent widths, so that flux calibration was not necessary. The blue region covered the range $3800-4100 \AA$, and flux calibration of the Ca II data followed the empirical transformations of Linsky et al. (1979), as described in equations (1-5) of Paper I. In particular, the flux $(\log F)$ in a $50 \AA$ region centred on $3950 \AA$ is given by

$\log F=8.199-3.585\left(0.082 E_{B-V}+0.682(B-V)\right)$. 
The mean colour excess $E(B-V)=0.02 \mathrm{mag}$ for the Blanco 1 region (Westerlund et al. 1988).

\subsection{Spectroscopy}

We have obtained $\mathrm{H} \alpha$ region spectra for 114 cluster members in this survey, of which 13 had too weak an equivalent width to measure (see Table 1 ). Of the remaining 101 spectra, 23 were in common with Paper I, so that, in total, we have accumulated $\mathrm{H} \alpha$ spectra of 125 stars of the 230 in the cluster survey of Abraham de Epstein \& Epstein (1985). Figure 1 shows the variation of $\mathrm{H} \alpha$ with $(B-V)$, where we have included the solar neighbourhood compilation of Panagi \& Mathioudakis (1993) on the diagram as we make comparisons later.

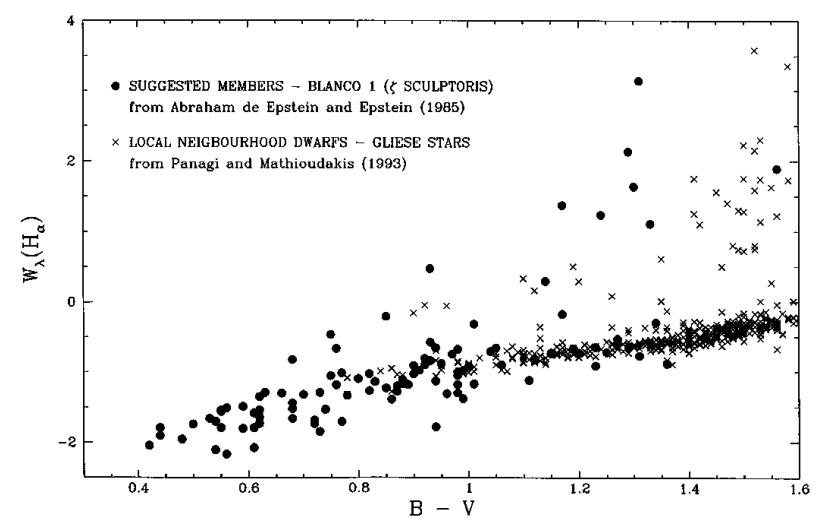

Fig. 1. Blanco 1 and the solar neighbourhood $\mathrm{H} \alpha$ variation with $(B-V)$. The lack of Blanco 1 observations beyond $(B-V)$ $\geq 1.4$ is due to target selection and not real

Of the 23 stars in common between this paper and Paper I, we find agreement to within $10 \%$ for the majority of $\mathrm{H} \alpha$ absorption equivalent widths. The exceptions are ZS 108, 109, which are about $50 \%$ and $100 \%$ greater here than in Paper I. We cannot comment precisely as to the discrepancy, except to say that we believe that there was no errors in the stellar positions during either observation. The sole $\mathrm{H} \alpha$ emission star, ZS 61, shows somewhat different behaviour. From Paper I, ZS 61 is the earliest spectral type to show emission (K2V-K3V), yet there was no discernible emission (or indeed absorption) in the present observations. Including the results of Paper I, we have covered some $60 \%$ of the cluster's K stars, of which we find 9 with $\mathrm{H} \alpha$ in emission. As the Abraham de Epstein \& Epstein study was extensive, it is therefore unlikely that many more $\mathrm{H} \alpha$ emitters remain to be discovered amongst the K star population.

Following Paper I we searched for the presence of lithium in those stars for which we had good quality medium resolution spectra around $\mathrm{H} \alpha$. By comparing our lithium measurements with those of other young clusters we hoped to find clues to the relative age of Blanco 1. Our previous study found 15 stars exhibited the Li I feature at $6707.8 \AA$. The present observations extend this list to 50 stars.

In addition to the $\mathrm{H} \alpha$ region, we have taken spectra of some 58 stars in $\mathrm{Ca} \mathrm{II}(\mathrm{K})$. The $30 \mathrm{~F}$ and $\mathrm{G}$ star spectra showed no core emission at our $S / N$, but of the remaining $28 \mathrm{~K}$ stars, we were able to identify six stars with measurable Ca II $(\mathrm{K})$. Four of the six $\mathrm{Ca} \mathrm{II}(\mathrm{K})$ emission stars, ZS 35, 37, 144 and 172 are also strong $\mathrm{H} \alpha$ emitters, whereas ZS 46 had too weak an $\mathrm{H} \alpha$ profile to measure. Not withstanding the poor seeing, the fact that the remaining $\mathrm{K}$ dwarfs, numbering 22 stars, did not show signs of Ca II emission cores is rather puzzling. This is not a problem of dispersion. Indeed, we have been able to measure $\mathrm{H} \alpha$ for nearly all the $\mathrm{K}$ dwarfs. Closer scrutiny reveals that 8 stars have possible errors in their positions, however, for all these stars we have been able to measure $\mathrm{H} \alpha$. If these stars possess chromospheres, then we must expect to see $\mathrm{Ca} \mathrm{II}(\mathrm{K})$ in emission at these effective temperatures. Alternatively, these stars may be field giants, in which the Ca II emission will be considerably weaker. We have decided to classify them as non-members, and they have been annotated accordingly in Table A2 (available electronically).

Based on a visual inspection and comparison with single star spectra of the same spectral type, we have found four new binaries in this survey, ZS 5, 14, 65 and 176, in addition to confirming that ZS 30a is a binary, based on its $\mathrm{H} \alpha$ profile. Including ZS 76, 80 and 120 found in Paper I, the total number of binaries is eight. We should note that auto-correlation of the spectra proved ambiguous.

The measured $\mathrm{H} \alpha$ and $\mathrm{Li} \mathrm{I}(6708)$ equivalent widths and $\mathrm{Ca} I \mathrm{I}(\mathrm{K})$ surface fluxes are presented in Table 1.

\subsection{Lithium}

Newly evolved clusters such as the Pleiades and $\alpha$ Persei are known to possess broad spreads in rotation rate, chromospheric activity and lithium abundance (Soderblom et al. 1993; Balachandran et al. 1988). It was therefore expected that Blanco 1 would exhibit similar patterns of lithium abundance. As part of our spectroscopic survey we searched for the $\mathrm{Li}$ I feature at $6707.8 \AA$ and were successful in measuring lithium abundances for 34 possible cluster members ( 4 of these were also measured in Paper I).

Figure 2 plots lithium equivalent width $\left(W_{\lambda}[\mathrm{Li}]\right)$ as a function of un-reddened colour $(B-V)_{0}$ for Blanco 1 and compares it to the Hyades, Pleiades and $\alpha$ Persei clusters. This figure is deliberately constructed to resemble the plots shown in Soderblom et al. (1993). The data for the $\alpha$ Persei cluster were taken from Balachandran et al. (1988), the Pleiades from Soderblom et al. (1993) and the Hyades from Soderblom et al. (1990). A large spread of $W_{\lambda}[\mathrm{Li}]$ is noticeable at any given spectral type for the Pleiades, $\alpha$ Persei and Blanco 1 clusters. The spread 
Table 1. Star No. assigned by Abraham de Epstein \& Epstein (1985), apparent $V$ magnitude, $(B-V)$ colour, equivalent width of $\mathrm{H} \alpha$ line (+ indicates emission), and equivalent width of Li I(6708) line. ? = Poor s/n, or too weak be measured. $\ddagger=$ no discernible $\mathrm{Ca} \mathrm{II}(\mathrm{K})$ emission

\begin{tabular}{|c|c|c|c|c|c|}
\hline $\begin{array}{c}\mathrm{ZS} \\
\mathrm{NO}\end{array}$ & $\begin{array}{c}V \\
\text { MAG }\end{array}$ & $\begin{array}{c}B-V \\
\mathrm{COL}\end{array}$ & $\begin{array}{c}W_{\lambda}[\mathrm{H} \alpha] \\
\mathrm{m} \AA\end{array}$ & $\begin{array}{c}W_{\lambda}[\mathrm{Li}] \\
\mathrm{m} \AA\end{array}$ & 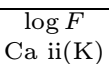 \\
\hline 1 & 13.48 & 0.94 & 1130 & $120 ?$ & \\
\hline 4 & 15.15 & 1.20 & 640 & 1102 & $\ddagger$ \\
\hline 5 & 15.52 & 1.40 & 570 & 902 & $\ddagger$ \\
\hline 6 & 15.78 & 1.35 & 530 & & $\ddagger$ \\
\hline 7 & 14.26 & 1.04 & 770 & & $\ddagger$ \\
\hline 8 & 14.98 & 1.35 & 640 & $240 ?$ & $\ddagger$ \\
\hline 10 & 13.15 & 0.83 & 1140 & & \\
\hline 12 & 13.88 & 1.31 & 460 & & $\ddagger$ \\
\hline 14 & 15.24 & 1.20 & 720 & & $\ddagger$ \\
\hline 15 & 15.19 & 1.34 & 510 & & $\ddagger$ \\
\hline 19 & 12.82 & 0.76 & 1190 & & \\
\hline 21 & 13.35 & 0.88 & 1170 & & \\
\hline 22 & 14.50 & 1.26 & 560 & & $\ddagger$ \\
\hline 23 & 15.72 & 1.36 & 2 & & $\ddagger$ \\
\hline 24 & 13.71 & 1.29 & 650 & & \\
\hline 25 & 12.83 & 0.96 & 1310 & & \\
\hline 28 & 15.69 & 1.29 & 860 & & $\ddagger$ \\
\hline 30 & 12.41 & 0.68 & 1450 & 1302 & \\
\hline $30 a$ & 12.74 & 0.76 & 670 & $120 ?$ & \\
\hline 31 & 12.43 & 0.75 & 470 & & \\
\hline 32 & 12.46 & 0.98 & 6802 & & \\
\hline 33 & 13.83 & 0.98 & 1180 & 602 & \\
\hline 34 & 12.82 & 0.72 & 1690 & & \\
\hline 35 & 14.42 & 1.14 & +290 & 60 & 5.40 \\
\hline 37 & 15.29 & 1.29 & +2130 & & 5.12 \\
\hline 42 & 14.14 & 1.17 & 200 & & 4.92 \\
\hline 46 & 14.00 & 1.17 & 2 & & 5.50 \\
\hline 47 & 14.38 & 1.24 & 900 & $190 ?$ & $\ddagger$ \\
\hline 48 & 10.72 & 0.48 & 1960 & & \\
\hline 56 & 11.17 & 0.54 & 1910 & & \\
\hline 58 & 12.15 & 0.68 & 970 & 120 & \\
\hline 61 & 13.51 & 0.93 & 2 & & \\
\hline 65 & 13.81 & 1.12 & 820 & & \\
\hline 72 & 13.40 & 0.85 & 1300 & & \\
\hline 75 & 12.79 & 0.94 & 620 & 90 & \\
\hline 78 & 12.99 & 0.98 & 1060 & & \\
\hline 79 & 12.38 & 0.98 & 1380 & & \\
\hline 80 & 13.64 & 0.86 & 2 & & \\
\hline 83 & 12.50 & 0.92 & 790 & 260 & \\
\hline 84 & 11.32 & 0.56 & 1410 & 100 & \\
\hline 88 & 13.81 & 1.16 & 2 & 902 & \\
\hline 90 & 10.62 & 0.50 & 1750 & 100 & \\
\hline 91 & 11.30 & 0.55 & 1500 & 402 & \\
\hline 95 & 12.34 & 0.92 & 920 & $150 ?$ & \\
\hline 98 & 12.02 & 0.99 & 1450 & & \\
\hline 100 & 12.49 & 0.88 & 1040 & & \\
\hline 102 & 12.50 & 0.77 & 1000 & & \\
\hline 105 & 11.42 & 0.53 & 1670 & 602 & \\
\hline 107 & 11.04 & 0.54 & 1620 & 1402 & \\
\hline 108 & 13.24 & 1.06 & 1090 & & \\
\hline 109 & 13.92 & 0.98 & 1380 & & \\
\hline 112 & 13.02 & 0.90 & 910 & 1602 & \\
\hline 113 & 12.92 & 0.86 & 1390 & & \\
\hline 118 & 12.98 & 1.04 & 670 & & \\
\hline 121 & 13.52 & 1.15 & 660 & & \\
\hline 122 & 12.57 & 0.80 & 1050 & & \\
\hline 124 & 13.62 & 0.93 & 840 & & \\
\hline 135 & 13.52 & 0.97 & 2 & 3102 & \\
\hline 141 & 11.96 & 0.68 & 1610 & & \\
\hline 143 & 14.98 & 1.37 & 490 & & $\ddagger$ \\
\hline 144 & 14.82 & 1.24 & +1230 & 502 & 5.39 \\
\hline 146 & 11.48 & 0.61 & 2080 & & \\
\hline 150 & 14.65 & 1.09 & 1020 & & $\ddagger$ \\
\hline 151 & 13.03 & 0.87 & 1400 & 602 & \\
\hline 153 & 12.38 & 0.73 & 1290 & & \\
\hline 154 & 13.36 & 0.95 & 880 & $210 ?$ & \\
\hline 158 & 13.47 & 1.05 & 660 & & \\
\hline 160 & 11.26 & 0.55 & 1800 & $120 ?$ & \\
\hline 161 & 12.61 & 0.77 & 1710 & & \\
\hline 162 & 14.11 & 1.33 & 590 & & $\ddagger$ \\
\hline
\end{tabular}

\begin{tabular}{rrrrrr}
\hline ZS & \multicolumn{1}{c}{$V$} & $B-V$ & $W_{\lambda}[\mathrm{H} \alpha]$ & $W_{\lambda}[\mathrm{Li}]$ & $\log F$ \\
NO & MAG & COL & \multicolumn{1}{c}{$\mathrm{m} \AA$} & $\mathrm{m} \AA$ & $\mathrm{Ca} \mathrm{ii}(\mathrm{K}$ \\
\hline 165 & 12.40 & 0.90 & 1030 & 150 & \\
167 & 12.07 & 0.73 & 1850 & & \\
170 & 12.18 & 0.70 & 1330 & 120 & \\
172 & 15.44 & 1.33 & +1110 & & 4.88 \\
173 & 12.46 & 0.97 & 750 & 802 & \\
174 & 15.16 & 1.18 & 800 & 120 & \\
175 & 13.60 & 1.00 & 920 & & \\
176 & 11.65 & 0.61 & 1590 & & \\
178 & 14.63 & 1.18 & 2 & & \\
180 & 11.77 & 0.62 & 1740 & & \\
182 & 11.72 & 0.63 & 1300 & 1102 & \\
186 & 12.22 & 0.75 & 1060 & & \\
187 & 13.87 & 0.99 & 980 & & \\
188 & 15.45 & 1.33 & 560 & & \\
189 & 11.61 & 0.62 & 1650 & 1202 & \\
190 & 11.13 & 0.68 & 1530 & 90 & \\
193 & 15.70 & 1.33 & 2 & & \\
194 & 10.86 & 0.42 & 2050 & & \\
195 & 11.82 & 0.66 & 1310 & 1102 & \\
196 & 11.51 & 0.74 & 1540 & & \\
201 & 11.75 & 0.62 & 1360 & 2202 & \\
202 & 15.50 & 1.35 & 2 & & \\
204 & 14.96 & 1.44 & 2 & & \\
205 & 14.00 & 1.39 & 3902 & & \\
206 & 14.43 & 1.17 & +1370 & 502 & \\
211 & 11.92 & 0.55 & 1550 & & \\
212 & 14.53 & 1.30 & 2 & & \\
214 & 11.40 & 0.59 & 1810 & 502 & \\
215 & 14.97 & 1.30 & +1640 & & \\
216 & 15.66 & 1.33 & 2 & & \\
217 & 10.59 & 0.44 & 1910 & & \\
220 & 15.64 & 1.23 & 650 & & \\
225 & 15.58 & 1.25 & 7202 & & \\
228 & 13.24 & 0.87 & 1200 & 802 & \\
229 & 15.73 & 1.29 & 2 & & \\
234 & 13.04 & 0.91 & 980 & & \\
237 & 15.19 & 1.19 & 670 & & \\
239 & 12.50 & 0.89 & 1180 & & \\
240 & 13.74 & 0.94 & 1780 & & \\
243 & 13.02 & 0.82 & 1030 & 2002 & \\
244 & 15.62 & 1.36 & 880 & & \\
245 & 13.19 & 0.78 & 1340 & 902 & \\
252 & 15.12 & 1.23 & 910 & & \\
257 & 12.77 & 1.01 & 1170 & & \\
\hline & & & & & \\
\end{tabular}

appears greatest for the lower mass stars where lithium depletion occurs more rapidly, such that by the age of the Hyades lithium has become no longer detectable. It is interesting to note that, except for a few instances (e.g. $\left.0.6 \leq(B-V)_{0} \leq 0.9\right)$ the Blanco 1 stars follow the same pattern as the Pleiades and $\alpha$ Persei stars, which would imply a similar age.

Lithium is a coarse estimator of relative age, the process by which it is depleted is far from understood and it is questionable how much weight should be attributed to our results. It can separate the Hyades from the Pleiades, $\alpha$ Persei and Blanco 1, yet it cannot resolve the latter with certainty.

\section{Discussion: Cluster age and chromospheric activity}

\subsection{The colour-magnitude diagram and cluster age}

We constructed the colour-magnitude diagram using the photometry published by Abraham de Epstein \& Epstein (1985) and Westerlund et al. (1988). First we corrected for 
the mean reddening. The colour excesses for the cluster are small; we used a mean excess of $E(B-V)=0.02 \mathrm{mag}$ (Westerlund et al. 1988) and assumed a visual absorption $\left(A_{\mathrm{v}}\right)$ of 0.06 mag. Next we removed superimposed field stars. Besides photometry the usual criteria for deciding cluster membership are proper motion and radial velocity measurements. Unfortunately at present these measurements are only available for a few of the higher mass stars (e.g. Perry et al. 1978; Edvardsson et al. 1995), although an extensive proper motion survey of the cluster is currently being undertaken by Platais et al. (1996). We base our final selection of cluster members almost entirely on photometry and consider all the $\mathrm{K}$ dwarfs with no measurable Ca II emission to be non-members.

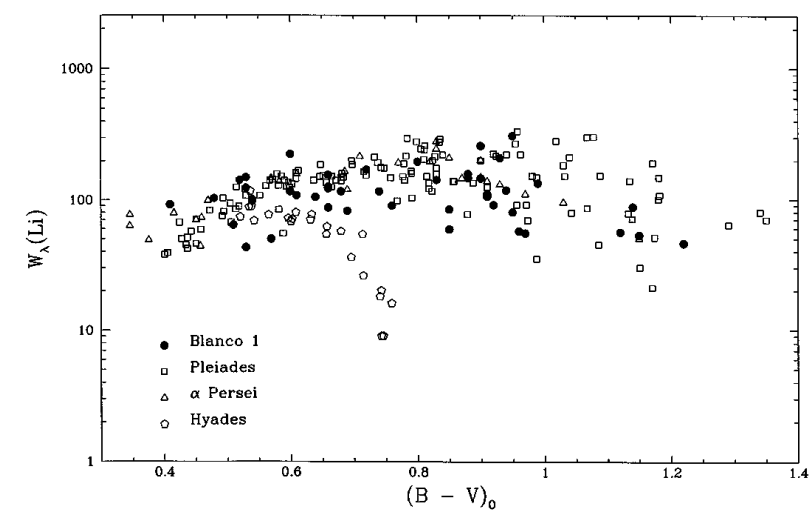

Fig. 2. Logarithm of the lithium equivalent width $\left(W_{\lambda}[\mathrm{Li}]\right)$ as a function of un-reddened colour $(B-V)_{0}$ for Blanco 1 as compared to the Hyades, Pleiades and $\alpha$ Persei clusters

Our revised list of cluster members with their photometry and positions is given in Table A1. This data was used to construct the colour-magnitude diagram for Blanco 1 (Fig. 3). We have superimposed the ZAMS of Lang (1991), assuming a distance modulus $(m-M)_{0}=6.96 \mathrm{mag}$ (Paper I). The diagram exhibits no turn-off and little if any evolution from the main-sequence. A gap is evident in the main-sequence between $(B-V)_{0} \approx 0.08 \mathrm{mag}$ and $0.27 \mathrm{mag}$. This been noted by other authors (e.g. Eggen 1970; Abraham de Epstein \& Epstein 1985) and is a common feature of young open clusters.

Our final list of non-members is presented in Table A2. Many of these stars were rejected by previous authors whilst the others all lie well above the ZAMS and are either field giants or nearby dwarfs. Abraham de Epstein \& Epstein (1985) suggested the latter could possibly be premain-sequence (PMS) stars still in a state of contraction to the ZAMS. This is unlikely given that even low-mass stars $\left(0.5 M_{\odot}\right)$ reach the vicinity of the ZAMS only after a few million years (e.g. Mihalas \& Binney 1981). Moreover this feature is not seen for the $\alpha$ Persei cluster (age $50 \mathrm{Myr}$ ), except perhaps for the very cool M-dwarfs (see

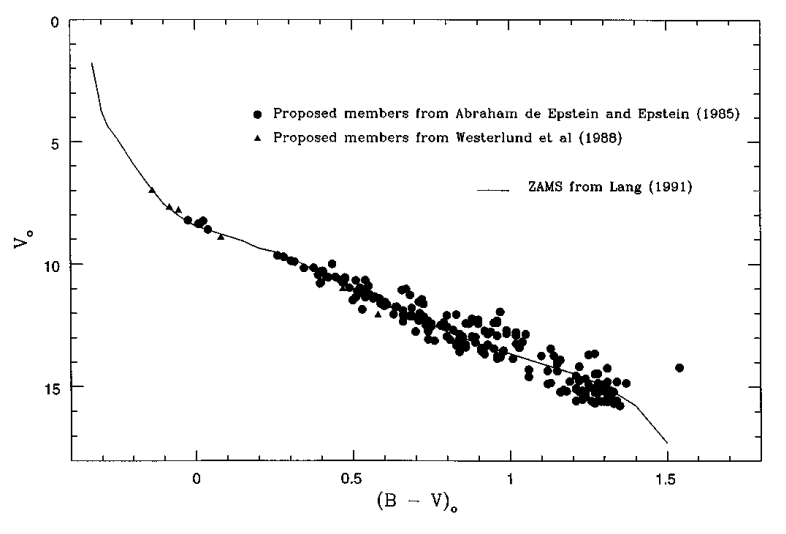

Fig. 3. Blanco 1 colour-magnitude diagram constructed from our revised list of cluster members given in Table A1. The ZAMS line is taken from Lang (1991)

Prosser 1992). In order to justify the existence of PMS stars, the cluster would have to be very young indeed. In fact we have removed virtually all the stars that deviated by more than 2 magnitudes from the lower portion of the ZAMS $(B-V>0.6 \mathrm{mag})$ and included them in our list of non-members as these are clearly field stars. We recognise that the width of our main-sequence still remains very large (given that the standard 0.75 mag spread is due to binarity), and therefore our membership list needs to be refined further to remove the remaining field star contaminants.

The lack of an obvious turn-off is indicative of a very young age and has lead previous authors to estimate ages $\leq 50$ Myr: e.g. $30 \mathrm{Myr}$ (Westerlund 1963), $20-40 \mathrm{Myr}$ (Eggen 1970), 45 Myr (Edvardsson et al. 1995), $50 \mathrm{Myr}$ (Epstein 1968; Perry et al. 1978; Abraham de Epstein \& Epstein 1985; Westerlund et al. 1988). Typically the cluster is believed to belong to the same age group as the Pleiades (Harris 1976). Throughout this paper we adopt the absolute ages of Mermilliod (1981) placing the age of the Pleiades at $\sim 70 \mathrm{Myr}$ and the $\alpha$ Persei cluster at $\sim 50$ Myr. Therefore on the basis of previous estimates, before considering our new age measuring technique, we can tentatively assume an age of 50-70 Myr for the Blanco 1 cluster.

A close examination of the upper main-sequence, however, reveals that all the latter age estimates hinge on the membership status of the B8 star HD 225187 (W71, Table A1), the bluest star on the colour-magnitude diagram. Perry et al. (1978) note that the proper motion of this star exceeds their criterion for cluster membership yet they include it in their final list of cluster members. The proper motion study of Platais et al. (1996) does not include this star as a member. Finally it is interesting to note that Perry et al. (1978) suspect HD 225187 to be a spectroscopic binary on the basis of its variable radial velocity. Recent studies (e.g. Kazarovets \& Samus 1990) 
show the star to be slightly variable. The existence of a companion star cannot be ruled out.

Figure 4 compares the upper main-sequence of the Pleiades and $\alpha$ Persei clusters with Blanco 1, where the turn-off from the main-sequence is clearly visible for both the Pleiades (age $70 \mathrm{Myr}$ ) and $\alpha$ Persei clusters (age $50 \mathrm{Myr}$ ). The dichotomy caused by HD 225187 (W71, Table A1) is clearly apparent. If we include the star on the colour-magnitude diagram then its position with respect to the ZAMS imposes a very young age on Blanco 1 (i.e. $\leq 50 \mathrm{Myr}$ ). If we remove this star then ages comparable and older than the Pleiades become feasible. It is conceivable that the lack of a turn-off is not necessarily a feature caused by a young age but could also be due to a deficiency of high-mass stars.

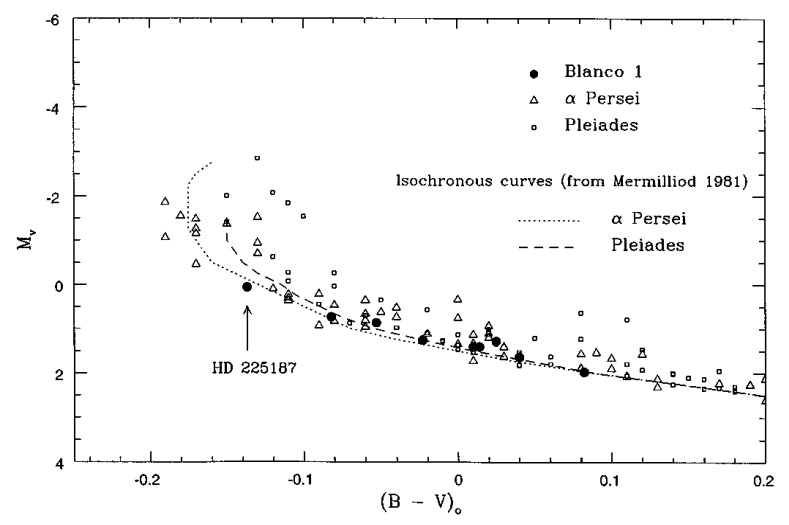

Fig. 4. Colour-magnitude for the clusters Blanco 1, $\alpha$ Persei and the Pleiades. The dashed lines show the isochronous curves for $\alpha$ Persei and the Pleiades (from Mermilliod 1981). The arrow indicates the B8 star HD 225187

\subsection{Ho and cluster age}

The lack of apparent evolution on the colour-magnitude diagram of Blanco 1 clearly supports a young age (Sect. 3.1). If we consider the age of the cluster to be $50 \mathrm{Myr}$, comparable with the age of $\alpha$ Persei, then we must expect the low-mass stars of the cluster to have a similar rotational velocity distribution and to exhibit a similar proportion of $\mathrm{K}$ dwarfs showing $\mathrm{H} \alpha$ in emission.

There exists a definite correlation between $\mathrm{H} \alpha$ emission and rotation. Stauffer \& Hartmann (1987) have shown that to $(R-I)_{\mathrm{K}}=0.7,((B-V) \sim 1.45)$ the more rapidly rotating K-dwarfs of the Pleiades cluster deviate from the general trend, showing consistently greater $W_{\lambda}[\mathrm{H} \alpha]$. This is consistent with the hypothesis that activity increases with rotation. Greater activity implies that either the mean chromosphere is hotter or that there is more surface inhomogeneity, both giving rise to increased $\mathrm{H} \alpha$ emission (Paper I).
Figure 5 shows the Blanco $1 \mathrm{H} \alpha$ emission equivalent width as a function of $(B-V)$, compared with other young clusters and the field. The rotational velocity distributions for the Pleiades and $\alpha$ Persei clusters are well known (e.g. Stauffer 1991). Following Stauffer \& Hartmann (1987) we crudely split these stars into fast and slow rotators to highlight the correlation between activity and rotation. A cursory examination of the plot shows that the Blanco 1 stars are distributed in the same manner as the Pleiades. Of interest, however, is the distribution of the young rapidly rotating $\alpha$ Persei stars on this plot, none showing the high levels of emission exhibited by the Pleiades and Blanco 1. Assuming these stars generate their magnetic flux by a shell dynamo process, then their high rotation rates should generate more magnetic flux, more chromospheric heating and hence stronger $\mathrm{H} \alpha$ emission. This appears not to be the case and may reflect a saturation of magnetic surface activity (e.g. O'Dell et al. 1995) that acts to suppress the magnetic-heating mechanism in the very rapid rotators. Also, this may be evidence that magnetic heating processes are not entirely responsible for the observed chromospheric emission seen in late-type stars.

The strongest $\mathrm{H} \alpha$ emitting Pleiades star HII 1883, $\left(W_{\lambda}[\mathrm{H} \alpha]=5.5 \AA\right)$ has a projected equatorial velocity of $v \sin i=140 \mathrm{~km} / \mathrm{s}$ (Stauffer et al. 1984). Similarly HII 347 , with $W_{\lambda}[\mathrm{H} \alpha]=2.8 \AA$, has a $v \sin i=75 \mathrm{~km} \mathrm{~s}^{-1}$ (Stauffer \& Hartmann 1987). At equatorial velocities of $75 \mathrm{~km} / \mathrm{s}$, the line broadening would be at least $1.6 \AA$, well above our resolution. No Blanco 1 stars were observed to have such broad $\mathrm{H} \alpha$ profiles, so we expect that the rotation distribution of Blanco 1 not to peak at such high velocities characterised by young clusters such as $\alpha$ Persei or the Pleiades. Nevertheless, it is likely that ZS $142\left(W_{\lambda}[\mathrm{H} \alpha]=3.14 \AA\right)$ and ZS $37\left(W_{\lambda}[\mathrm{H} \alpha]=2.13 \AA\right)$, the two strongest $\mathrm{H} \alpha$ emitters are relatively rapid rotators amongst the Blanco 1 population itself.

Figure 5 reveals that the onset of $\mathrm{H} \alpha$ emission in Blanco 1 is at $(B-V)=0.93(\mathrm{ZS}$ 61), whereas the older solar neighbourhood dwarfs (taken from Panagi \& Mathioudakis (1993)) show an onset of emission at $(B-V)=1.10$. Similarly the Pleiades and $\alpha$ Persei clusters also exhibit an onset of $\mathrm{H} \alpha$ emission earlier than the older field stars (Prosser et al. 1991). Studies of these and other young clusters have shown a correlation between $W_{\lambda}[\mathrm{H} \alpha]$ and spectral type and a trend toward $\mathrm{H} \alpha$ emission at early spectral types. Prosser et al. (1991) compared the $\mathrm{H} \alpha$ emission strengths of selected M-dwarfs for the Pleiades, Hyades and field. They found that the mean strength of $\mathrm{H} \alpha$, on average, declines as one passes from the Pleiades through the Hyades to the field, and therefore may act as a proxy for age. This can also be seen for the K-dwarfs.

Figure 5 shows that the distribution of Blanco $1 \mathrm{~K}$ dwarfs is similar to the Pleiades yet considerably different from the Hyades and field. This is indicative of the youth 


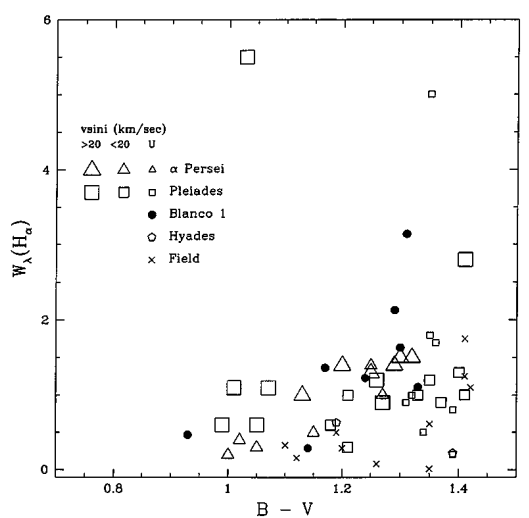

Fig. 5. $\mathrm{H} \alpha$ emission equivalent width as a function of $(B-V)$ for Blanco 1 compared with other young clusters and the field. The $\alpha$ Persei and Pleiades stars are crudely split into fast and slow rotators to highlight the correlation between activity and rotation. U indicates unknown rotational velocity. Note that the distribution of Blanco 1 stars is very similar to that of the Pleiades

of Blanco 1 and the Pleiades. In a study of the chromospheric activity of low-mass members in the Hyades, Stauffer et al. (1991) noted that the calibration of this effect could yield a new tool for the determination of the relative ages of open clusters.

In order to quantify the decline of $\mathrm{H} \alpha$ emission as an age indicator, the ratio of $\mathrm{H} \alpha$ emitters to the total number of observed cluster members $\left(N_{\mathrm{em}} / N_{\text {tot }}\right)$ within the spectral range $0.8 \leq(B-V) \leq 1.4$ was calculated for a selection of "reference" clusters of differing ages. The spectral range was chosen to cover only the $\mathrm{K}$ dwarfs, the region where a strong link exists between the activity-age and rotation-age relations.

Table 2. Cluster, the fraction of $\mathrm{H} \alpha$ emitters amongst $\mathrm{K}$ dwarfs $(0.8 \leq(B-V) \leq 1.4)$ and the age, assuming published ages for all clusters. The age of Blanco 1 has been interpolated from Fig. 6

\begin{tabular}{ccc}
\hline Cluster & $N_{\mathrm{em}} / N_{\text {tot }}$ & AGE $($ Myrs.) \\
\hline$\alpha$ Persei & 0.218 & 50 \\
Pleiades & 0.157 & 70 \\
Blanco 1 & 0.121 & 90 \\
Hyades & 0.014 & 600 \\
\hline
\end{tabular}

The ratio $\left(N_{\text {em }} / N_{\text {tot }}\right)$ is plotted as a function of age for the "reference" clusters in Fig. 6 . The points were well fitted by an exponential function therefore we show $\ln \left(N_{\mathrm{em}} / N_{\text {tot }}\right)$ versus $\ln$ (Age). The straight line was fitted to the $\alpha$ Persei, Pleiades and Hyades points. The values $\left(N_{\text {em }} / N_{\text {tot }}\right)$ were attributed poissonian errors and dashed lines added to approximate its upper and lower bounds. No uncertainty was assumed for the ages. The ages of the
Pleiades, $\alpha$ Persei and Hyades clusters were all taken from Mermilliod (1981). The ratio $\left(N_{\mathrm{em}} / N_{\mathrm{tot}}\right)$ for the three "reference" clusters and Blanco 1 are shown against their respective ages in Table 2 .

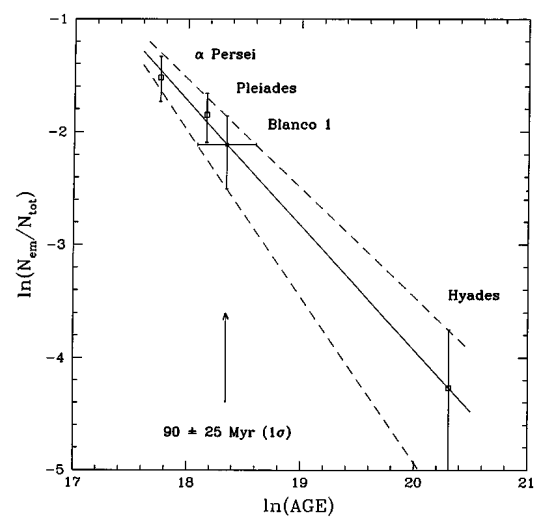

Fig. 6. Logarithmic plot of the ratio of $\mathrm{H} \alpha$ emitters to the total number of observed cluster members $\left(N_{\mathrm{em}} / N_{\mathrm{tot}}\right)$ as a function of age for $\alpha$ Persei, the Pleiades and Hyades. The plot allows us to estimate the age of Blanco 1 (note the $\approx 1 \sigma$ uncertainty shown by the horizontal error bar)

The "reference" clusters have all been subject to extensive spectroscopic surveys. The Pleiades and Hyades have been popular targets for many years, in the main, due to their close proximity to the solar neighbourhood. In particular, comprehensive searches have been made for $\mathrm{H} \alpha$ emission lines amongst the late-type populations of these clusters (e.g. Prosser et al. 1991). It is therefore unlikely that many more $\mathrm{H} \alpha$ emitters remain to be discovered, particularly amongst the relatively bright K-dwarfs. The $\left(N_{\mathrm{em}} / N_{\mathrm{tot}}\right)$ values are therefore to be regarded as extremely reliable and unlikely to change significantly.

Although the cluster Blanco 1 has not been studied to the extent of the Pleiades, $\alpha$ Persei and Hyades it is nevertheless apparent that the cluster contains relatively few $\mathrm{H} \alpha$ emitters. This spectroscopic survey has examined $\sim 60 \%$ of the Abraham de Epstein \& Epstein (1985) sample in the $0.8 \leq(B-V) \leq 1.4$ spectral range. The estimated value of $\left(N_{\text {em }} / N_{\text {tot }}\right)$ is therefore considered realistic for the entire K-dwarf population of the cluster. Plotting this value in Fig. 6 reveals that the cluster is closer in age to the Pleiades than $\alpha$ Persei and is considerably older than previous estimates. An estimate of $\sim 90 \mathrm{Myr}$ was extracted from the plot, with a $68 \%$ confidence interval of $\pm 25 \mathrm{Myr}$. We recognise that this estimate is likely to change once cluster membership becomes better defined. Preliminary membership information from the proper motion study of Platais et al. (1996) of a number of K dwarfs has allowed us to obtain a lower estimate of $60 \mathrm{Myr}$ for the age of the cluster. 


\subsection{Chromospheric activity}

\subsection{1. $\mathrm{H} \alpha$ and rotation}

We consider here how $\mathrm{H} \alpha$ emission and absorption act as proxies for stellar rotation. Looking at Fig. 5, the variation of $\mathrm{H} \alpha$ emission against $(B-V)$, amongst the $\mathrm{K}$ dwarfs, for the four clusters and the field. There is a large spread in equivalent width, much more than for the $\mathrm{H} \alpha$ absorption stars (Fig. 1). H $\alpha$ emission is more sensitive to rotation and stellar effective temperature, for $\mathrm{K}$ dwarfs than G dwarfs. The sensitivity is due to the contrast between the chromospheric and photospheric temperatures, as at higher effecive temperatures greater chromospheric electron densities (and hence temperatures) are needed to drive $\mathrm{H} \alpha$ into emission (Cram \& Mullan 1979; Panagi 1994). Once in emission, $\mathrm{H} \alpha$ emission has an approximate cubic dependency on the chromospheric electron density, over the region of its formation. Thus, although $\mathrm{H} \alpha$ in emission indicates rapid rotation, its high sensitivity to atmospheric conditions makes intepretation of the rotation distribution very difficult.

This is not so for $\mathrm{H} \alpha$ absorption, whose general trend as a function of $(B-V)$ is shown for both Blanco 1 and the solar neighbourhood dwarfs relationship in Fig. 1. The narrower relative spread, when compared to the $\mathrm{H} \alpha$ emission at a given $(B-V)$, is consistent with $\mathrm{H} \alpha$ absorption being much less sensitive to variations in chromospheric temperature and density.

We now consider the importance of the scatter in absorption equivalent widths at a given $(B-V)$. Two, perhaps complementary, explanations can be used to interpret this spread, assuming that the stars exhibit a spread in rotation rates. Equating increased rotation rate with increased surface activity, we would expect that the faster rotators will show increased levels of surface inhomogeneity, implying that local variations in temperature and density would dominate the $\mathrm{H} \alpha$ profile. This is the filling-in scenario, and so increased rotation in this case equates to decreasing (in magnitude) equivalent width. Alternatively (or in tandem), increased rotation could yield an overall greater mean chromospheric temperature (and density), so that the $\mathrm{H} \alpha$ absorption equivalent width could actually increase in magnitude. These conclusions follow naturally from the theoretical work by Cram \& Mullan $(1979,1985)$ on the formation of $\mathrm{H} \alpha$ in cool stars. They showed that, for a given effective temperature, increasing the chromospheric temperature gradient initially gives rise to a greater $\mathrm{H} \alpha$ absorption, before, at some critical chromospheric temperature (and hence density), $\mathrm{H} \alpha$ is driven into emission.

\subsubsection{The zero $\mathrm{H} \alpha$ stars}

The transition from $\mathrm{H} \alpha$ absorption to emission predicted by Cram \& Mullan (1979) is an intriguing observational problem. One would expect to see stars with apparently strong chromospheres, evidenced by strong Ca II emission, but little or no $\mathrm{H} \alpha$. They have been termed the zero- $\mathrm{H} \alpha$ stars. A small number have indeed been observed in the solar neighbourhood (Robinson et al. 1990; Mathioudakis \& Doyle 1992). Mathioudakis \& Doyle (1992) also looked at $\mathrm{Mg}$ II emission in a number of late-type solar neighbourhood dwarfs, and found five stars with generally weak emission. These stars have $(B-V) \geq 1.2$ and have also been termed $\mathrm{d} M(\mathrm{e}), \mathrm{d} K(\mathrm{e})$ or zero- $\mathrm{H} \alpha$ stars. Thus two sub-classes of zero $\mathrm{H} \alpha$ stars have been identified, weak and strong chromosphere stars. In a Mg II emission with colour diagram, Mathioudakis \& Doyle (1992) observed that the hottest $\mathrm{d} K(\mathrm{e})$ or $\mathrm{d} M(\mathrm{e})$ stars have fluxes that lie intermediate between those that show either $\mathrm{H} \alpha$ emission or absorption, and so argued that they are in fact active stars with strong chromospheres. They similarly argued that the cooler $\mathrm{d} M(\mathrm{e})$ stars are in fact quite close to radiative equilibirum, with little or no signs of a chromosphere. The results are based on the computatons by Cram \& Mullan (1979) who have shown that radiative equilibrium models of late $\mathrm{K}$ to mid-M dwarfs produce decreasing $\mathrm{H} \alpha$ absorption, ranging from $0.24 \AA$ to $0.08 \AA$. The parallel between $\mathrm{Mg}$ II emission and Ca II emission can be drawn by noting that there exists a strong linear relationship between the two, for stars in the solar neighbourhood (Panagi \& Mathioudakis 1993). Based on their relatively strong $\mathrm{Ca}$ II emission, we would therefore expect the Blanco 1 stars ZS $46(\sim \mathrm{K} 5)$ and ZS $61(\sim \mathrm{K} 2)$ (Table 1) to be active stars, and their lack of a measurable $\mathrm{H} \alpha$ absorption feature implying that filling-in of the $\mathrm{H} \alpha$ core by active region emission dominates the line profile. Hence they would prove to be extremely useful in the study of chromospheric physics, as $\mathrm{H} \alpha$ could flip between absorption and emission under small changes in chromospheric temperature and density.

An interesting suggestion has been put forward by Bopp (1985) and Saar (1992) regarding the evolutionary status of the $\mathrm{d} K(\mathrm{e})$ and $\mathrm{d} M(\mathrm{e})$ stars. They suggested that these stars are marginal BY Dra stars, rapidly rotating binaries, showing both a high level of surface inhomogeneity, manifest in stellar spots, and showing strong chromospheric emission in $\mathrm{H} \alpha$. They also argue that the $\mathrm{d} K(\mathrm{e})$ and $\mathrm{d} M(\mathrm{e})$ stars are in a short evolutionary phase between the $\mathrm{H} \alpha$ emission stars and the $\mathrm{H} \alpha$ stars, brought about as the stars are rotationally braked; the slower rotating stars have weaker chromospheres and so show $\mathrm{H} \alpha$ in absorption. There is no discussion of the time-scale of this evolutionary phase. Based on this scenario, and given the relatively young age of Blanco 1, we would expect to see this transitional phase in $\mathrm{K}$ dwarfs, should it exist, whereas in the much older solar neighbourhood dwarfs the effect would be seen only for the late $\mathrm{K}$ and $\mathrm{M}$ dwarfs. The two stars ZS 46 and 61 also seem to satisfy this criteria. 


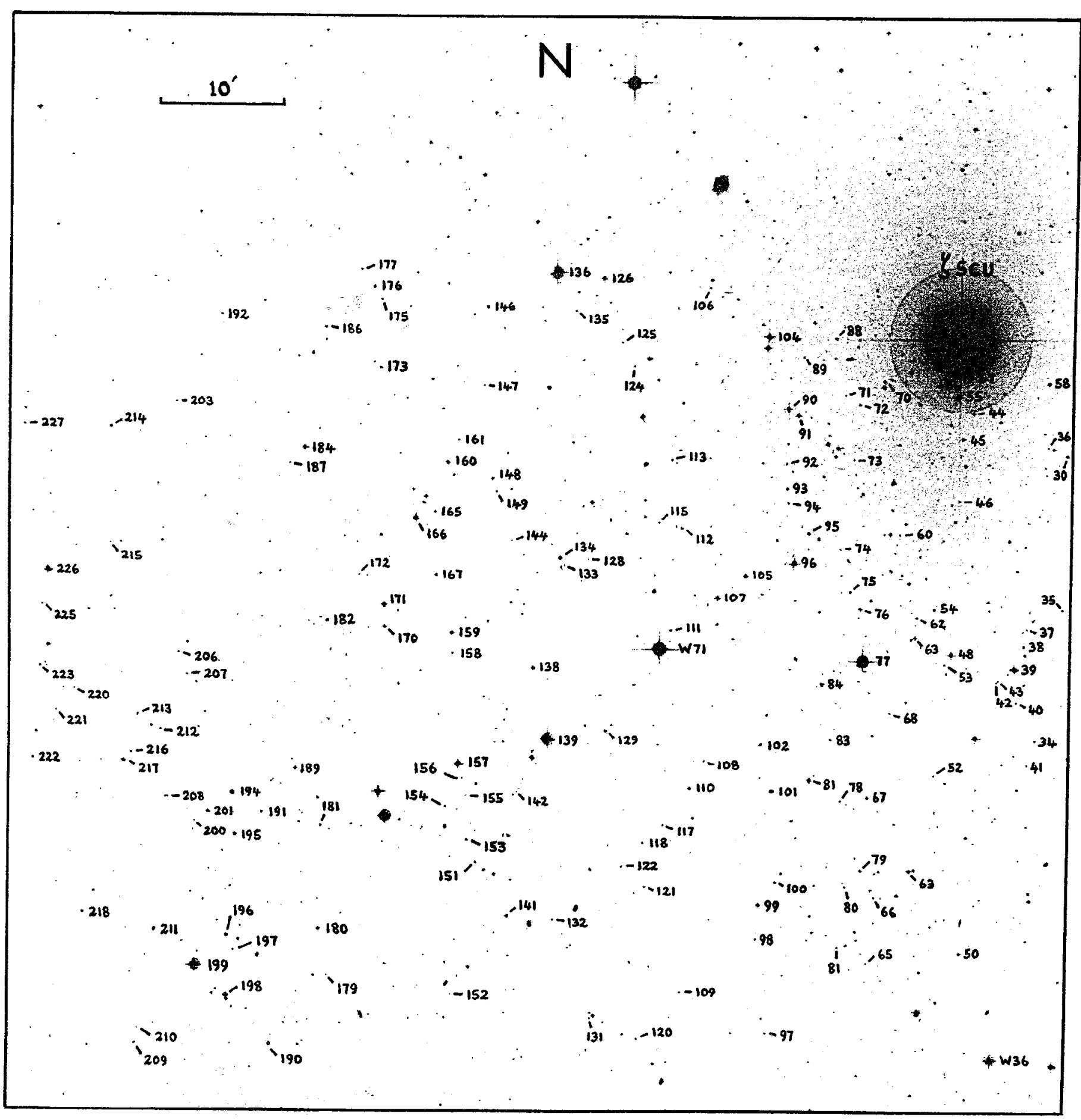

Fig. 7. A 4-frame composite digitized sky survey map of the Blanco 1 region centred near ZS 134 , with $\mathrm{RA}=00^{\mathrm{h}} 02^{\prime} 16.05^{\prime \prime}$ and DEC $=-30^{\circ} 17^{\prime} 38.6^{\prime \prime}$. Approximately $80 \%$ of the cluster members of Table A1 are shown 


\section{Summary}

We have presented new spectroscopy of the open cluster Blanco 1, primarily examining the $\mathrm{G}$ and $\mathrm{K}$ dwarf population which make up the vast majority of published photometric observations. We have revised cluster membership based on available photometry and spectroscopy, and have included accurate positions for all these stars. We recognise that our memership list is still contaminated by field stars. Cluster membership will be improved when the proper motion survey of Platais et al. (1996) is published.

By quantifying the number of $\mathrm{H} \alpha$ emitters within the cluster's K dwarf population we obtain a new age estimate for the cluster of $90 \pm 25 \mathrm{Myr}$. This is substantially greater than previous estimates based on isochrone fitting to the main-sequence turn-off. We believe this dichotomy may be explained by a paucity of high-mass stars and doubt as to the position of the bluest star on the CM diagram. This new age measuring technique is found to be more sensitive than lithium, and its use is recommended for young clusters, where a strong correlation exists between activity-rotation and age.

Acknowledgements. We would like to thank Charles Prosser for making available all his compiled data on $\alpha$ Persei, the Pleiades and Hyades clusters. Many thanks also to Imants Platais who very kindly supplied us with preliminary information from his recent proper motion survey which is expected to be published shortly. We thank Mike Irwin for his help with the APM, also Martin Hendry, Andrew Cameron and John Stauffer for useful comments on the statistics and interpretation of our data. Finally we wish to thank the staff at the AAT, particularly Raylee Stathakis, for help during the observations.

\section{References}

Abraham de Epstein A.E., Epstein I., 1985, AJ 90, 1211

Balachandran S., Lambert D.L., Stauffer J.R., 1988, ApJ 333, 267

Blanco V.M., 1949, PASP 61, 183

Bond H.E., 1972, PASP 84, 583

Bopp B.W., 1985, in: Zeilik M., Gibson D.M. (eds.) "Cool stars, stellar systems and the Sun". Springer, Berlin

Cram L.E., Mullan D.J., 1979, ApJ 234, 579

Cram L.E., Mullan D.J., 1985, ApJ 294, 626

Dolidze M.V., 1959, Astron. Circ. USSR 200, 9

Edvardsson B., Petterson B., Kharrazi M., Westerlund B., 1995, A\&A 293, 75

Eggen O.J., 1970, ApJ 161, 159

Eggen O.J., 1972, ApJ 173, 63

Epstein I., 1968, AJ 73, 556

Harris G.L.H., 1976, ApJS 30, 451

Howarth I.D., Murray J., Mills D., 1994, Starlink User Note 50.15
Kazarovets E.V., Samus N.N., 1990, IAU Inform. Bull. Var. Stars 3530, 1

Irwin M., McMahon R., 1992, Gemini No. 37, RGO, Cambridge Lang K.R., 1991, Astrophysical Data. Springer, New York Berlin Heidlelberg

Lawden, M., 1995, Starlink User Note, 1.3

Linsky J.L., Worden S.P., McClintock W., Robertson R.M., 1979, ApJ 41, 47

Lyngå G., Wramdemark S., 1984, A\&A 132, 54

Mathioudakis M., Doyle J.G., 1992, A\&A 262, 523

Mermilliod J.-C., 1981, A\&A, 97, 235

Mihalas D., Binney J., 1981, Galactic Astronomy (2nd ed.) Freeman, New York

O'Dell M.A., 1995, D. Phil thesis, University of Sussex, Brighton

O'Dell M.A., Panagi P., Hendry M.A., Collier Cameron A., 1995, A\&A 294, 715

Panagi P.M., Mathioudakis M., 1993, A\&AS 100, 343

Panagi P.M., 1994, in: 1st Conf. on the Wilson-Bappu effect and related topics, Freire-Ferrero R. (ed.) Strasbourg, France

Panagi P.M., O'Dell M.A., Collier Cameron A., Robinson R.D., 1994, A\&A 292, 439 (Paper I)

Perry C.L., Walter D.K., Crawford D.L., 1978, PASP 90, 81

Phelps R.L., Janes K.A., 1993, AJ 106, 1870

Platais I., Kozhurina-Platais V., Barnes S., Horch E.P., 1996, AJ (in preparation)

Prosser C.F., 1995 (private communication)

Prosser C.F., 1992, AJ 103, 488

Prosser C.F., Stauffer J.R., Kraft R.P., 1991, AJ 101, 1361

Robinson R.D., Cram L.E., Giampapa M.S., 1990, ApJS 74, 891

Sadler E., Harrison S., Lee S., 1991, AAO Observers' Guide, 4th edition (AAO:UM 1.4)

Salpeter E.E., 1955, ApJ 121, 161

Saar S., 1992, in: Giampapa M.S. \& Bookbinder J. (eds.) Cool star workshop, ASP Conf. 26, p. 288

Skumanich A., 1972, ApJ 171, 565

Soderblom D.R., Oey M.S., Johnson D.R.H., et al., 1990, AJ 99, 595

Soderblom D.R., Jones B.F., Balachandran S., et al., 1993, AJ 106, 1059

Stauffer J.R., 1991, in: Catalano S., Stauffer J.R. (eds.) Angular Momentum Evolution of Young Stars. Kluwer Academic Publishers, Dordrecht, p. 117

Stauffer J.R., Hartmann L.W., Soderblom D.R., et al., 1984, ApJ 280, 202

Stauffer J.R., Hartmann L.W., 1986, ApJS 61, 531

Stauffer J.R., Hartmann L.W., 1987, ApJ 318, 337

Stauffer J.R., Giampapa M.S., Herbst W., et al., 1991, ApJ 374, 142

Westerlund B.E., 1963, MNRAS 127, 183

Westerlund B.E., Garnier R., Lundgren K., Petterson B., Breysacher J., 1988, A\&AS 76, 101 